\title{
Embolia gasosa por dióxido de carbono durante uma hepatectomia laparoscópica: relato de caso
}

\section{Gas embolism by carbon dioxide during a laparoscopic hepatectomy: case report}

Juliana Rosa Meloํ. Fernanda Paula Cavalcante². Flávio Lobo Maia³. Lorena Antônia Sales Vasconcelos ${ }^{4}$. Inara Nobre de Castro ${ }^{1}$.

1 Médica residente do Programa de Anestesiologia, Universidade Federal do Ceará (UFC), Fortaleza, Ceará, Brasil. 2 Titulo Superior em Anestesiologia pela Sociedade Brasileira de Anestesiologia, Médica Anestesiologista responsável pelo Centro de Ensino e Treinamento (CET) e preceptora do Programa de Anestesiologia, Hospital Universitário Walter Cantídio (HUWC), Fortaleza, Ceará, Brasil. 3 Titulo Superior em Anestesiologia pela Sociedade Brasileira de Anestesiologia, Médico Preceptor do Programa de Anestesiologia, Hospital Universitário Walter Cantídio (HUWC), Fortaleza, Ceará, Brasil. 4 Médica Preceptora do Programa de Anestesiologia, Hospital Universitário Walter Cantídio (HUWC), Fortaleza, Ceará, Brasil.

\section{RESUMO}

A cirurgia laparoscópica tem se tornado uma técnica cada vez mais frequente em nosso meio, porém, não está isenta de complicações. Embora rara, a embolia gasosa por $\mathrm{CO}_{2}$ é uma complicação grave, associada a uma mortalidade de até $28 \%$. Esse é um relato de caso de um paciente do sexo masculino, 73 anos, que foi submetido a uma hepatectomia parcial videolaparoscopica para o tratamento de um adenocarcinoma. Após 200 minutos de cirurgia apresentou instabilidade hemodinâmica, diminuição da saturação de oxigênio, acidose respiratória e diminuição da $\mathrm{ETCO}_{2}$. Foi então desfeito o pneumoperitoneo e o paciente foi tratado com drogas vasoativas. O rápido diagnostico da embolia por $\mathrm{CO}_{2}$ foi importante para a rápida recuperação e ausência de sequelas no paciente.

Palavras-chave: Embolia gasosa. Dióxido de carbono. Laparoscopia.

\section{ABSTRACT}

Laparoscopic surgery has become an increasingly frequent technique in our midst. But it is not free from complications. Although rare, $\mathrm{CO}_{2}$ gas embolism is a serious complication associated with a mortality rate of up to $28 \%$. This is a case report of a male patient, 73 years old, who underwent a partial videolaparoscopic hepatectomy for the treatment of an adenocarcinoma. After 200 minutes of surgery he presented hemodynamic instability, decreased oxygen saturation, respiratory acidosis and decreased $\mathrm{ETCO}_{2}$. The pneumoperitoneum was then disassembled and the patient was treated with vasoactive drugs. The rapid diagnosis of $\mathrm{CO}_{2}$ embolism was important for a rapid recovery and absence of sequelae in the patient.

Keywords: Gas embolism. Carbon dioxide. Laparoscopy.

Autor correspondente: Juliana Rosa Melo, Avenida da Abolição, 4043, apartamento 402, Mucuripe, Fortaleza, Ceará. CEP: 60165-082.Telefone: +55 85 98801-7450. E-mail: juliana.melorosa@gmail.com

Conflito de interesses: Não há qualquer conflito de interesses por parte de qualquer um dos autores.

Recebido em: 20 Fev 2017; Revisado em: 11 Abr 2017; Aceito em: 11 Abr 2017. 


\section{INTRODUÇÃO}

A técnica cirúrgica laparoscópica vem se tornando cada vez mais frequente em nosso meio, uma vez que diminui a agressão cirúrgica e o tempo de internação hospitalar. Para ser feita insufla-se dióxido de carbono $\left(\mathrm{CO}_{2}\right)$ na cavidade para uma melhor visualização e manipulação cirúrgica. Essa distensão causada pelo gás pode causar inúmeras complicações, dentre elas a embolia por $\mathrm{CO}_{2} \cdot{ }^{1}$ Essa complicação é causada por entrada de $\mathrm{CO}_{2}$ em uma veia, artéria ou órgão sólido danificado. ${ }^{2}$ Está associada a hipotensão, cianose, hipercarbia ou hipocarbia, arritmia e colapso cardíaco devido a um aprisionamento de gás na veia cava e no átrio direito. Ocorre uma diminuição do retorno venoso e débito cardíaco. O padrão ouro para o diagnóstico é a detecção de $\mathrm{CO}_{2}$ no átrio ou ventrículo direito, porém devido a sua rápida eliminação o diagnóstico pode ser baseado em parâmetros fisiológicos.

\section{CASO CLÍNICO}

Paciente do sexo masculino, pardo, 73 anos, $86 \mathrm{~kg}$, com diagnóstico de hepatocarcinoma. A cirurgia proposta seria uma hepatectomia parcial por via laparoscópica. Paciente referia hipertensão arterial sistêmica e diabetes melitos. Os exames pré-operatórios estavam normais. A anestesia iniciou-se por uma peridural a nível de T12-L1 com $10 \mathrm{ml}$ de ropivacaina $0,2 \%+2 \mathrm{mg}$ de morfina, a indução feita com sufentanil (25mcg), propofol (120mg) e cisatracúrio (7mg). Paciente colocado em ventilação mecânica com os seguintes parâmetros: Volume Corrente: $600 \mathrm{ml} / \mathrm{min}$, Frequência Respiratória: 12, Ri:e 1:2, PEEP: $5 \mathrm{~cm} \mathrm{H}_{2} \mathrm{O}$. A manutenção da anestesia foi feita com sevoflurano a $2 \%$. Puncionado acesso venoso central em veia jugular interna direita e monitorização da pressão arterial invasiva na artéria radial esquerda. Após 200 minutos de pneumoperitôneo, paciente apresentou hipotensão arterial $(80 \times 50 \mathrm{mmHg})$ não responsiva a etilefrina, queda da saturação de $\mathrm{O}_{2}$ para $92 \%$. Iniciado noradrenalina $0,5 \mathrm{mcg} / \mathrm{kg} / \mathrm{min}$. Uma gasometria foi feita com os seguintes resultados: $\mathrm{pH}: 7,13$ $\mathrm{PO}_{2}: 81,8 \quad \mathrm{PCO}_{2}: 102,4 \mathrm{HCO}_{3}: 33,6 \quad \mathrm{SatO}_{2}: 91,4 \%$. Nesse momento o cirurgião foi avisado da suspeita diagnostica e o pneumoperitôneo foi desfeito. Paciente foi reposicionado em cefalo declive e decúbito lateral esquerdo. Após 40 minutos da retirada do pneumoperitôneo o paciente já não necessitava de droga vasoativa, $\mathrm{SatO}_{2}$ havia se normalizado, nova gasometria arterial mostrava $\mathrm{pH}: 7,35 \mathrm{PO}_{2}: 100 \mathrm{PCO}_{2}: 42 \mathrm{HCO}_{3}: 22,5$ $\mathrm{SatO}_{2}: 97,3 \%$. Após o fim da cirurgia paciente foi encaminhado a UTI pós-operatória onde foi extubado sem intercorrência.

\section{DISCUSSÃO}

Apesar de ser uma complicação rara, a incidência de embolia causado por $\mathrm{CO}_{2}$ pode variar de acordo com o método de detecção utilizado para o diagnóstico. A mortalidade pode chegar a $28 \% .^{3}$ A ecografia transesofágica vem sendo utilizada para diagnosticar a embolia por $\mathrm{CO}_{2}$. Lin, et $\mathrm{al}^{4}$ identificou embolia em 69 de 403 pacientes (17,1\%) durante a retirada endoscópica da veia safena para a realização da revascularização miocárdica. Embolia mínima foi vista em $53(13,1 \%)$, moderada em $14(3,5 \%)$ e massiva em $2(0,5 \%)$.
Kim, et $\mathrm{al}^{5}$ reportou uma incidência de embolia por dióxido de carbono durante histerectomia laparoscópica utilizando a ecografia transtorácica. Algum grau de embolia foi observado em todas as pacientes, em 37,5\% a quantidade de bolhas observadas foi mais da metade do diâmetro do átrio direito e ventrículo direito. Nenhuma paciente sofreu instabilidade hemodinâmica ou mudanças no eletrocardiograma. A maioria dos casos graves de embolia ocorrem pelo posicionamento da agulha de Veress diretamente dentro de uma veia. Nas embolias tardias as causas prováveis são entrada do ar por meio de vasos abertos na parede abdominal ou no sitio cirúrgico. ${ }^{56}$ Mayer, et al, ${ }^{7}$ descreveu uma mortalidade de $60 \%$ em uma infusão continua a $1,2 \mathrm{ml} / \mathrm{kg} / \mathrm{h}$ de gás, o que equivale a $72 \mathrm{ml} / \mathrm{min}$ em uma pessoa de $60 \mathrm{~kg}$. Esse volume é apenas $5 \%$ do volume que pode ser feito em uma veia canulada por uma agulha de Veress em um minuto. Os sintomas iniciais podem ser hipotensão sistêmica, cianose, dispneia, bradicardia ou taquicardia. Pode ocorrer um aumento rápido da $\mathrm{ETCO}_{2}$ seguido de sua queda. A embolia pode progredir para colapso cardiovascular e redução do fluxo sanguíneo pulmonar. $\mathrm{O}$ efeito do aprisionamento do gás causa obstrução de saída do ventrículo direito, diminuição do retorno venoso, falência de ventrículo esquerdo e ventrículo direito, embolia paradoxal e colapso cardiovascular (Quadro 1).

Quadro 1. Alterações fisiológicas, sinais e sintomas da embolia por dióxido de carbono.

\begin{tabular}{|c|c|c|}
\hline Causa & Alterações fisiológicas & Sinais e sintomas \\
\hline \multirow{2}{*}{$\begin{array}{c}\text { Dificuldade de } \\
\text { troca gasosa }\end{array}$} & Diminuição da $\mathrm{PO}_{2}$ & Dispneia \\
\cline { 2 - 3 } & Diminuição da $\mathrm{PaCO}_{2}$ & Cianose \\
\cline { 2 - 3 } & Diminuição do $\mathrm{pH}$ & Hipoxia \\
\hline \multirow{2}{*}{$\begin{array}{c}\text { Aumento da } \\
\text { pós-carga de VD }\end{array}$} & Aumento da PAP & Hipotensão \\
\cline { 2 - 3 } & Cor pulmonale & Dores no peito \\
\cline { 2 - 3 } & Falência de VD & Sopro cardíaco \\
\hline \multirow{2}{*}{$\begin{array}{c}\text { Diminuição do } \\
\text { enchimento de VE }\end{array}$} & Fiminuição do debito & Arritimias \\
\cline { 2 - 3 } & Parada cardíaca & Mudanças no ECG \\
\cline { 2 - 3 } & Morte \\
\hline
\end{tabular}

VD: ventriculo direito; VE: ventriculo esquerdo; PAP: pressão da artéria pulmonar; ECG: eletrocardiograma.

Fonte: Park EY, Kwon JY, Kim KJ, Carbon Dioxide Embolism during Laparoscopic Surgery. Yonsei Med J. 2012;53(3):459-66.

Um estudo feito com ecocardiográfica transesofágica identificou embolia gasosa após a injeção de $0,02 \mathrm{ml}$ de gás e foi 50 a 100 vezes mais sensível a presença de embolia do que a $\mathrm{ETCO}_{2}{ }^{8}$ Estudos mostraram a identificação de embolia em 11 de 16 pacientes submetidos a colecistecomia laparoscópica (68\%). ${ }^{9}$ A melhor posição do probe é na veia cava inferior, pois reduz a chance de encontrar fluxo turbulento no átrio direito 
por injeção de mediações. As desvantagens da ecocardiográfica transesofágica são alto custo, complexidade técnica e ser um exame operador dependente. Couture, et al, ${ }^{10}$ demonstrou que independente do modo de administração do dióxido de carbono (bolus ou infusão) o primeiro sinal foi a diminuição da $\mathrm{ETCO}_{2}$. A prevenção pode ser feita com correto posicionamento da agulha de Veress, uso de pressões baixas de insuflação do gás. ${ }^{3}$ A manutenção da pressão venosa central maior que a pressão intrabdominal pode reduzir o risco de embolia. O trendelemburg reverso diminui a incidência de embolia. Na suspeita de embolia gasosa por dióxido de carbono o pneumoperitôneo deve ser desfeito. A ventilação deve ser feita com $\mathrm{FiO}_{2}$ de $100 \%$, para melhorar a relação ventilação perfusão. A hiperventilação irá aumentar a excreção de $\mathrm{CO}_{2}$. Aumento da pressão venosa central com expansão de volume pode dificultar a nova entrada de gás na veia lesada. A posição da mesa cirúrgica deve ser modificada para trendelemburg e decúbito lateral esquerdo, essa posição faz com que as bolhas sigam para o ápice do

\section{REFERÊNCIAS}

1. Smith HJ. Carbon dioxide embolism during pneumoperitoneum for laparoscopic surgery: a case report. AANA J. 2011;79(5):371-3.

2. Park EY, Kwon JY, Kim KJ. Carbon dioxide embolism during laparoscopic surgery. Yonsei Med J. 2012;53(3):459-66.

3. Hong JY, Kim WO, Kil HK. Detection of subclinical carbon dioxide embolism by transesophageal echocardiography during laparoscopic radical prostatectomy. Urology. 2010;75(3):581-4.

4. Lin TY, Chiu KM, Wang MJ, Chu SH. Carbon dioxide embolism during endoscopic saphenous vein harvesting in coronary artery by-pass surgery. J Thorac Cardiovasc Surg. 2013;126(6):2011-5.

5. Kim CS, Kim JY, Kwon JY, Choi SH, Na S, An J, et al. Venous air embolism during total laparoscopic hysterectomy: comparsion to total abdominal hysterectomy. Anesthesiology. 2009;111(1):50-4

6. Gutt CN, Oniu T, Mehrabi A, Schemmer P, Kashfi A, Kraus T, et al. Circulatory and Respiratory Complications of Carbon Dioxide Insufflation. Dig Surg. 2004;21:95-105. coração e não entrem nas artérias pulmonares. Caso o paciente esteja com acesso venoso central, a aspiração pode ajudar no diagnóstico e tratamento. A causa mais importante de morte é a falência cardíaca direita causada pelo aprisionamento das bolhas no ventrículo direito, vasoconstrição pulmonar e consequentemente falência de ventrículo esquerdo. Pode-se usar vasopressores e agentes inotrópicos para manutenção do débito cardíaco. Vasodilatadores podem ser usados para diminuir a vasoconstricção pulmonar e reduzir a pós carga de VD.

\section{CONCLUSÃO}

É de vital importância que o anestesiologista e o cirurgião estejam cientes do risco da embolia por $\mathrm{CO}_{2}$ durante uma cirurgia laparoscópica. $\mathrm{O}$ reconhecimento rápido dos sintomas e o início do tratamento irá diminuir a morbi-mortalidade dos pacientes.

7. Mayer KL, Ho HS, Mathiesen KA, Wolfe BM. Cardiopulmonary responses to experimental venous carbon dioxide embolism. Surg Endosc. 1998;12(8):1025-30.

8. O’Sullivan DC, Micali S, Averch TD, Buffer S, Reyerson T, Schulam $\mathrm{P}$, et al. Factors involved in gas Embolism after laparoscopic injury to inferior vena cava. J Endourol. 1998;12(2):149-54.

9. Landercasper J, Miller GJ, Strutt PJ, Olson RA, Boyd WC. Carbon dioxide embolization and laparoscopic cholecystectomy. Surg Laparosc Endosc. 1993;3(5):407-10.

10. Couture P, Boudreault D, Derouin M, Allard M, Lepage Y, Girard $\mathrm{D}$, et al. Venous carbon dioxide embolism in pigs: an evaluation of end-tidal carbon dioxide, transesophageal echocardiography, pulmonary artery pressure, and precordial auscultation as monitoring modalities. Anesth Analg. 1994;79(5):867-73.

11. Cottin V, Delafosse B, Viale JP. Gas embolism during laparoscopy: a report of seven cases in pacients with previous abdominal surgical history. Surg Endosc. 1996;10(2):166-9.

\section{Como citar:}

Melo JR, Cavalcante FP, Maia FL, Vasconcelos LA, Castro IN. Embolia gasosa por dióxido de carbono durante uma hepatectomia laparoscópica: relato de caso. Rev Med UFC. 2017 set-dez;57(3):70-72. 